\title{
Mohs Surgery for Basal Cell Carcinoma: A Systematic Review
}

\author{
Ariadna Miranda Alvarado Benítez ${ }^{\mathbf{1}}$, Erika Bautista Rodríguez ${ }^{\mathbf{1}}$, Alberto Robles Méndez \\ Hernández $^{2 *}$, Rodrigo Dominic Cerqueda Audirac ${ }^{1}$, Laura Paulina Baena Hernández ${ }^{1}$, Lourdes \\ Rocío Álvarez López ${ }^{1}$, Andrés Armendariz Rodríguez ${ }^{1}$ \\ ${ }^{l}$ Medical intern, Universidad Nacional Autónoma de México, Mexico city, Mexico \\ ${ }^{2}$ Department of General Surgery, Hospital Angeles Metropolitano, Mexico city, Mexico
}

*Corresponding Author: Alberto Robles Méndez Hernández, Department of General Surgery, Hospital Angeles Metropolitano, Mexico city, Mexico.

\begin{abstract}
Introduction: Basal cell carcinoma (BCC) is the most common type of nonmelanoma skin cancer (80\% of all skin cancers), and the most commonin humans. Mohs surgery is highly effective at removing skin cancer because the edges of the removed cancer tissue are checked much more thoroughly under the microscope than with regular surgery. These BCCs are preferably treated with Mohs micrographic surgery $(M M S)$ which minimizes the risk of recurrence and avoids unnecessary removal of healthy tissue
\end{abstract}

Objective and Methods: The bibliographic recollection was carried through two search engines: PubMed and Cochrane Library.

The following criteria were the ones to be fulfilled for the results to be included in the hereby revision: antiqueness not older than 10 years, and Spanish or English language. After reviewing the analysis methodology and quality of the articles, 13 articles from PubMed, and 7 from the Cochrane Library were included.

Conclusions: Mohs micrographic surgery is, currently, the gold standard for the cleavage of a wide variety of basal cell carcinomas, with a lower tumor recurrence when compared with conventional surgery. In the United States of America, the primary basal cell carcinoma's recurrence rate is $1 \%$, while for the recurrent one is $5.6 \%$.

Keywords: Basal cell carcinoma, Mohs Micrographic Surgery.

\section{INTRODUCTION}

Keratinocyte carcinomas (KCs) are, by far, the most common malignancies worldwide and exceed the prevalence of all other cancers combined. Traditionally, approximately $80 \%$ of $\mathrm{KC}$ cases have been attributed to basal cell carcinoma (BCC) and 20\% to squamous cell carcinoma (SCC). However, recent studies point to an increasing SCC incidence relative to $\mathrm{BCC}$, moving the historical $4: 1$ ratio to 2.5:1 or even closer. [1]

Basal cell carcinoma (BCC) is the most common malignant tumor of the skin. It is also the most common cancer in humans in some countries. BCC is a malignant neoplasm derived from nonkeratinizing cells, it has its origin in the basal layer of the epidermis. The histology of the tumor and the surrounding stroma is characteristic.
Basal cell carcinoma was first described in 1824 by Jacob who called it "ulcusrodens"; its current nomenclature was proposed by Krompecher in 1903. It is the most common type of nonmelanoma skin cancer $(80 \%$ of all skin cancers) and the most common malignancy in humans. It is originated from the basal layer of the epidermis or pluripotent adnexal basaloid cells and it is almost always seen in the areas that are usually exposed to the sun, and hairy parts of the skin. [2]

Mohs micrographic surgery is, currently, the gold standard for the cleavage of a wide variety of basal cell carcinomas. [3]

\section{OBJECTIVE AND METHODS}

\subsection{Objective}

Perform a bibliographic review about Mohs surgery for basal cell carcinoma through the PubMed and Cochrane Library search engines 
to synthesize the information that has previously been published and highlight the benefits it has when compared to conventional surgeries.

\subsection{Justifying}

Synthesizing and organizing the published information in order to reach a conclusion about Mohs surgery's indications and objectives, as well as its impact on patients who have been diagnosed with basal cell carcinoma.

\subsection{Methodology}

The bibliographic recollection was performed in two search engines: PubMed and Cochrane Library.

In PubMed, two searches were performed; the words used in the first process were Mohs surgery And Basal cell carcinoma; using "And" with the Boolean Operator function. The used filter was 10 years, and this search gave 842 results.

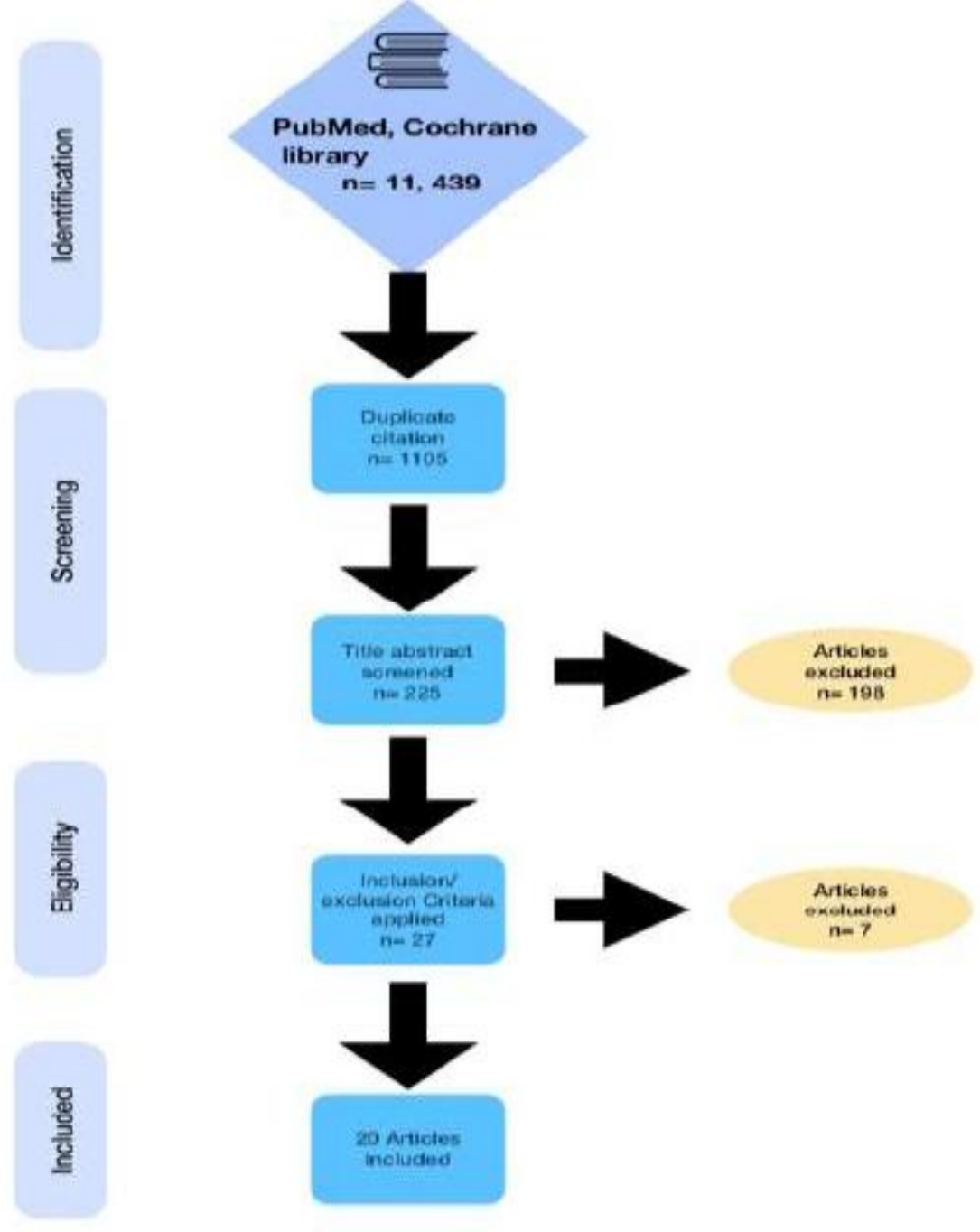

Figure1. PRISMA Diagram

For the second search, the used words were Basal-cell carcinoma surgery, and the filter was 10 years, giving 10,523 results. From the first search, 11 results were selected, while from the second one, two results fulfilled the following inclusion criteria, which are included in the PRISMA model. (Figure 1):

- Clear methodology
- Stablished objective

- No conflicts of interest

- More than three authors

- Concluding results

On Cochrane Library the words used were Mohs surgery And Basal cell carcinoma, "And" with the Boolean Operator function. 
The filters used for this search were: Title abstract and keyword, and antiqueness not higher than 10 years.

This search gave 74 results: 71 Trials and 3 Cochrane reviews, from which seven articles that fulfilled the following inclusion criteria, included in the PRISMA model, were included. (Figure 1):

- Clear methodology

- Stablished objective

- No conflicts of interest

- More than three authors

- Concluding results

- Spanish or English language

\subsection{Definition}

Basal cell carcinoma is the most common malignant neoplasm of epithelial origin, its incidence has raised in the past couple years around 4 to $8 \%$ per year. It is located predominantly in the head and the neck, it presents a slow growth, and rarely metastasizes, but it can produce local destruction and compromise widespread tissue, cartilage, and bone areas. [4]

Throughout history, the basal cell carcinoma has been considered a common malignant neoplasm among old age patients. However, a higher incidence in people younger than 40 years old has recently been observed. This can be attributed to the change in lifestyles and environmental interactions.

The risk of suffering basal cell carcinoma has been associated with being sunburnt during childhood, skin cancer family history, the usage of tanning beds, chronic immunosuppression, [5] photosensitizing drugs,[6] ionizing radiation, and exposure to carcinogenic chemical substances, especially arsenic, however, the main environmental risk factor is exposure to ultraviolet radiation.

In 2018, a multicentric study reported young Spanish American patients who were diagnosed with basal cell carcinoma, which was found more frequently in women, due to higher exposure to tanning beds, and recreational activities, as well as hormonal factors. These findings can also have its origin in the derivation bias since it is possible for women to get attended for cosmetic reasons. [7]
In the same study, it was reported that Caucasian people are affected more frequently, as well as in individuals that present the Fitzpatrick I and II phototypes, however, it has been mistakenly said that a dark skin could have certain "immunity". This fact has resulted in late diagnosis, and therefore, unfavorable results in the treatment.

\subsection{Topography}

In terms of location, $48 \%$ of the tumors were found in the nasal area, followed by the periorbital area, with $18 \%$. DOI: 10.20431/2456-0022.0501001. Comparing with another prospective study, the anatomical distribution was found to be as follows: nasal area $48 \%$, cheeks $14 \%$, forehead $7 \%$, the lower part of the eyelid $7 \%$, temple $6 \%$, upper lip and inner canthus 3\%, outer canthus 3\%, chinandears 2\% (Figure 2).[3]

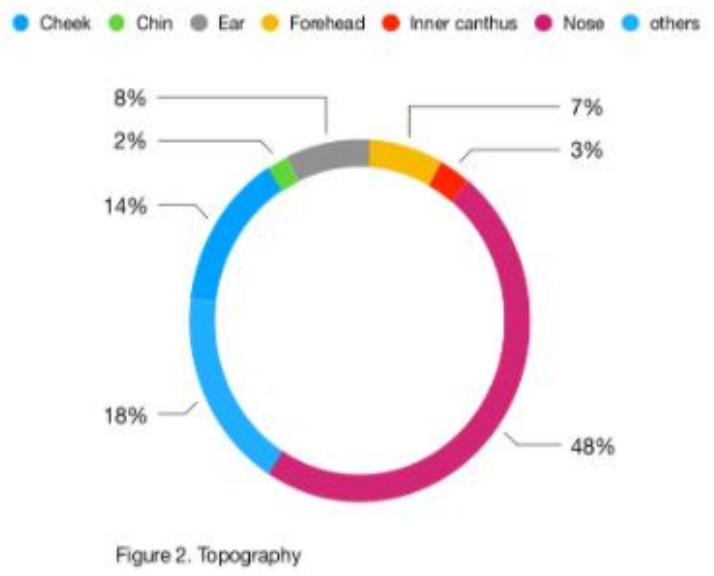

Figure2. Topography

Speaking about recurring tumors, $62.1 \%$ had been previously treated through conventional surgeries, and $13.8 \%$ with Mohs surgeries, which shows us the high effectiveness of the Mohs surgery when compared with the first one.

\subsection{Mohs Surgery}

Mohs is the last name of the person who invented the technique: Frederic Mohs, MD. Mohs surgery is highly effective at removing skin cancer because the edges of the removed cancer tissue are checked much more thoroughly under the microscope than with regular surgery.[8] These BCCs are preferably treated with Mohs micrographic surgery (MMS) which minimizes the risk of recurrence and avoids unnecessary removal of healthy tissue.[9]

There are three goals in the treatment for BCC: 
(1) To excise the tumor completely so that there is no recurrence of tumor at a later time, (2) To avoid any functional impairment resulting from excision of the tumor,

(3) To provide the best possible cosmetic outcome, especially for the lesions that are on the face. [10]

Mohs surgery is not necessary for all skin cancers, but it is useful when:

1. The location of the skin cancer is near areas that are important for daily activities, like the fingers, or for appearance, like the nose;

2. Earlier treatments have not worked;

3. A tumor is large; and

4. Regular surgery is less likely to remove cancer.

Mohs surgery has long high long-term healing rates, compared to other treatment modes, therefore, it is the gold standard for the basal cell carcinoma's cleavage, especially in recurring and high-risk $\mathrm{CBC}$.

The main reasons why people underwent Mohs surgery were: incomplete tumor cleavage with a $15 \%$, recurrence with an $11 \%$, skin preservation with a $10 \%$, and a hard anatomical site with an 8\%.[3]

Different authors evaluated in a prospective study the resection requirements and reconstruction techniques of a group of patients diagnosed with basal cell carcinoma and compared the surgical result of Mohs surgery with that expected from the conventional cleavage and the reconstruction for the same lesions.

From the studied patients and those who underwent a conventional cleavage, the following results were obtained: $47 \%$ obtained suboptimal reconstructions, for a $24 \%$ the histological margins were incomplete, for a $20 \%$ there were no changes, that's to say, the patients would get the same results from both techniques, and for a percentage that is lower than $9 \%$, but not less important, the patients would've lost fundamental structures.

\subsection{Surgical Technique}

Mohs surgery is typically performed under local anesthesia in a nonsterile procedure room in the outpatient setting. The first step of the Mohs procedure is to identify the biopsy site. After confirming the site, the patient must settle in a reclined and covered position. Using a marker, the tumor must be delineated with a margin of $1-5 \mathrm{~mm}$ (depending on its extension), afterwards, the area must be cleaned up with $4 \%$ chlorhexidine gluconate, except for the periocular area, where instead, $7.5 \%$ or $10 \%$ povidone-iodine must be used. After that, local anesthesia with lidocaine and $1 \%$ or $2 \%$ epinephrine must be used. Once anesthetized, the surgeon may or may not make use of curettage for detecting tumoral extensions that may not be clinically visible. An incision is then made with a scalpel (most typically a \#15 blade) along the outlined margin, beveled at a 30 to 45 angle, and the tissue is then sharply excised to an appropriate depth along a flat horizontal plane. Once removed, the orientation of the specimen should be vigilantly maintained in preparation for mapping. Hemostasis is then most commonly achieved with electrosurgery, electrocautery, chemical cautery, or direct pressure. Finally, the patient is temporarily bandaged and instructed to wait for 0.5 to 2.0 hours in a comfortable area during the mapping, slide preparation, and histopathology interpretation. https://doi.org/10.20431/24560022.0501001

\subsubsection{Necessary Surgical Margins for Basal Cell Carcinomas Treated with Micrographic Mohs Surgery}

Previous studies have demonstrated that the patient's tumor and its characteristics can predict a greater amount of stages that are necessary to be able to achieve clear margins.

In a retrospective analysis of 295 cases of basal cell carcinoma that were treated with the Mohs surgery, the tumor and its histological characteristics were related to the necessary margins for complete removal of the tumor.

The following results were found: The patients who were included were 137 women and 102 men with an average age of 64 years. The most common histologic subtype was the nodular one, followed by the infiltrative one.[11]

It is worth highlighting that in the Spanish American study that featured young patients, the same results regarding the most common histological subtype were reported, represented in the following way: nodular con a $37.5 \%$, followed by infiltrative/trabecular with an $18.9 \%$, and the mixed one with a $16.2 \%$.[7]

For the tumor size, they were divided into 4 groups; group 1:<6 mm, group 2: 6 to $10 \mathrm{~mm}$, group 3: 11-19mm, and group 4:>19 $\mathrm{mm}$. 
Histologically, they were classified by nonaggressive (superficial and nodular) and aggressive (micronodular, infiltrative, morpheaform, and metatypical).

In the board, (figure 3) the necessary margins for achieving the full removal of the tumor for each group found in said study, are shown.

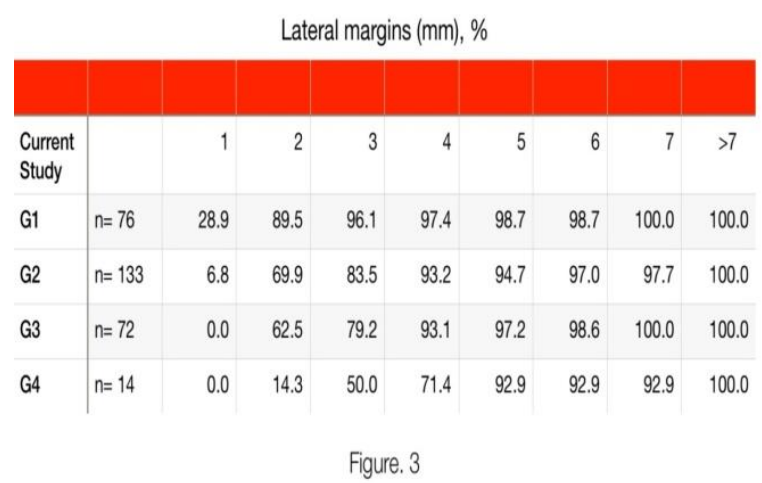

\subsubsection{Recurrence in the Mohs Surgery}

In a prospective study made in Spain about basal cell carcinoma's recurrence and survival in 534 patients after undergoing MMC, recurrence was found in 32 cases, of which 14 underwent a new Mohs intervention, 12 required an excision by other specialists, four received local radiotherapy, and one refused to receive another intervention. The reported recurrence rate in all of the tumors was $1.2 \%$ for primary $\mathrm{CBC}$ and $10.4 \%$ for previously treated CBC. In the United States, a recurrence of $1 \%$ for primary $\mathrm{CBC}$, and $5.6 \%$ for recurring $\mathrm{CBC}$ is reported. [9]

\section{Conclusions}

Based on the performed review, it is concluded that the keratinocyte carcinoma is the most common malignant neoplasm in all the world, and within this cancer lineage, the basal cell carcinoma is the most common skin malignant tumor $(80 \%$ of all skin cancer). In some countries, it is even the cancer type with the most incidence. It predominates in the head and neck, being found more frequently in the nasal area, followed by the preorbital area and the cheeks. Less frequently, it has been found in the forehead, the lower part of the eyelid, the upper lip, the inner canthus, the outer canthus, the chin, and the ears.

Likewise, it is concluded that the micrographic Mohs surgery, currently is the gold standard for the cleavage of a wide variety of basal cell carcinomas, with a lower tumor recurrence rate when compared to conventional surgery. Based on the consulted studies, this technique will help extirpate the tumor completely, so as to reduce the cancer recurrence rate, the post-surgical functional deterioration of the treated area, and have the best possible aesthetic results.

Mohs surgery is best indicated when the location of the cancer is in zones that are important for the daily activities that need the best cosmetic results in order to not alter the patient's appearance (fingers, nose, etc.), when the tumor is big, and when the tumor's excision through a conventional technique is less likely.

In the United States, the primary basal cell carcinoma's recurrence rate is $1 \%$, while for the recurring one, it's $5.6 \%$.

Lastly, a higher amount of studies in Mexico about the incidence of this kind of cancer need to be carried out, to be able to apply this surgical technique and evaluate the post-surgical results and its effectiveness regarding the treatment of this illness.

\section{REFERENCES}

[1] Rogers HW, Weinstock MA, Feldman SR, Coldiron BM. Incidence Estimate of Nonmelanoma Skin Cancer (Keratinocyte Carcinomas) in the US Population, 2012. JAMA Dermatol. 2015 Oct 1;151(10):1081.

[2] Tuzun Y, Kutlubay Z, Engin B, Serdaroglu S. Basal Cell Carcinoma. In: Xi Y, editor. Skin Cancer Overview [Internet]. InTech; 2011 [cited 2020 Sep 8]. Available from: http://www.intechopen.com/books/skin-canceroverview/basal-cell-carcinoma

[3] Wain RAJ, Tehrani H. Reconstructive outcomes of Mohs surgery compared with conventional excision: A 13-month prospective study. J Plast Reconstr Aesthet Surg. 2015 Jul;68(7):946-52.

[4] Lai V, Cranwell W, Sinclair R. Epidemiology of skin cancer in the mature patient. Clin Dermatol. 2018 Mar;36(2):167-76.

[5] Silverberg MJ, Leyden W, Warton EM, Quesenberry CP, Engels EA, Asgari MM. HIV Infection Status, Immunodeficiency, and the Incidence of Non-Melanoma Skin Cancer. JNCI J Natl Cancer Inst. 2013 Mar 6;105(5):350-60.

[6] Robinson SN, Zens MS, Perry AE, Spencer SK, Duell EJ, Karagas MR. Photosensitizing Agents and the Risk of Non-Melanoma Skin Cancer: A Population-Based Case-Control Study. J Invest Dermatol. 2013 Aug;133(8):1950-5.

[7] Aluma-Tenorio MS, Ávila-Álvarez A, Jaimes N, Caballero-Uribe N, González A, Terzian 
LR, et al. Basal cell carcinoma treated with Mohs micrographic surgery in young IberoAmerican patients. Int J Dermatol. 2018 Dec;57(12):1447-53.

[8] Etzkorn JR, Alam M. What Is Mohs Surgery? JAMA Dermatol. 2020 Jun 1;156(6):716.

[9] Català A, Garces JR, Alegre M, Gich IJ, Puig L. Mohs micrographic surgery for basal cell carcinomas: results of a Spanish retrospective study and Kaplan-Meier survival analysis of tumour recurrence. J Eur Acad Dermatol Venereol. 2014 Oct;28(10):1363-9.
[10] Quazi SJ, Aslam N, Saleem H, Rahman J, Khan S. Surgical Margin of Excision in Basal Cell Carcinoma: A Systematic Review of Literature. Cureus [Internet]. 2020 Jul 15 [cited 2020 Sep 8]; Available from: https://www.cureus.com/ articles/33368-surgical-margin-of-excision-inbasal-cell-carcinoma-a-systematic-review-ofliterature

[11] Cerci FB, Kubo EM, Werner B, Tolkachjov $\mathrm{SN}$. Surgical margins required for basal cell carcinomas treated with Mohs micrographic surgery according to tumor features. J Am Acad Dermatol. 2020 Aug;83(2):493-500.

Citation: Alberto Robles Méndez Hernández. Mohs Surgery for Basal Cell Carcinoma: A Systematic Review. ARC Journal of Dermatology. 2020; 5(1):1-6. DOI: https://doi.org/10.20431/2456-0022.0501001.

Copyright: (C) 2020 Authors. This is an open-access article distributed under the terms of the Creative Commons Attribution License, which permits unrestricted use, distribution, and reproduction in any medium, provided the original author and source are credited. 